\title{
ARTICLE OPEN \\ Cross-sectional study assessing the performance of the Arabic translated childhood asthma control test
}

\author{
Majid AlTeneiji ${ }^{1}$, Alia AlKalbani ${ }^{1}$, Huda Nasser ${ }^{1}$, Durdana Iram $^{1}$, Afaf Alblooshi $\mathbb{D}^{2}$ and Hassib Narchi ${ }^{2}$
}

The standard Arabic version of the Childhood Asthma Control Test (C-ACT) has never been previously evaluated in Arab countries. We studied its correlation in Arabic speaking children in the United Arab Emirates (UAE), with both the GINA assessment of asthma control and the resulting changes in asthma management. The Arabic C-ACT was completed by the children or by their parents when needed. A GINA based level of asthma control score was assigned by their managing physician. The correlation between the different cut- scores of the C-ACT and GINA were studied. A total of 105 eligible children with asthma (aged between 4 and 11.8 years, $61 \%$ boys) were enrolled. The Arabic translated C-ACT had a high reliability (Cronbach alpha $81 \%$ ) and validity (as it correlated well with the GINA level of control). We found that using it with the traditional cut-score of 19 overestimated the degree of asthma control. Instead, a calculated optimal cut-score of 20 estimated more accurately the level of asthma control as assessed both by the GINA assessment and also by changes in asthma management. The current Arabic version of the C-ACT has a good reliability and validity. By using a single optimal cut-point of 20 , it can be used to assess both the level of asthma control and of treatment control. It does not, however, accurately define asthma control when using the originally proposed cut-score of 19 . Physicians need to recognise that the C-ACT cut-points may vary in different populations. We suggest that cut-scores of translated versions need to be modified in different geographical settings.

npj Primary Care Respiratory Medicine (2018)28:41 ; doi:10.1038/s41533-018-0109-3

\section{INTRODUCTION}

Asthma is the most common chronic disease in children ${ }^{1}$ with an estimated prevalence of 6 to $13 \%$ in those aged between 6 and 14 years in the United Arab Emirates (UAE). ${ }^{2,3}$ It is a leading cause of school absenteeism when poorly controlled. ${ }^{4}$

The assessment of asthma control in children plays an important role in asthma management. It now replaces asthma severity to aid asthma management in the most recent Global Initiative for Asthma (GINA) guideline. ${ }^{5}$ Assessment of asthma control based on symptoms in children is challenging, as it is essentially subjective, depending on the reliability of the parent's perception of their child's airway obstruction, ${ }^{6}$ which may differ from the physician's perception of asthma control, which is also subjective. 7

Multiple questionnaires have already been introduced into clinical practice to aid the assessment of asthma control in children. One example is the Childhood Asthma Control Test (C$\mathrm{ACT}$ ) questionnaire, increasingly implemented in clinical settings for children between the ages of four and eleven years. ${ }^{8}$ It is completed by the parents or by the children themselves. The concordance between C-ACT and the GINA levels of asthma control is not always optimal. ${ }^{9,10}$

The C-ACT has been translated into different languages, ${ }^{11-14}$ including Arabic. The standard classical Arabic language is the official language that is formally taught, read, written and understood across the Arab world. However, as a multitude of only spoken dialects, often with different pronunciations, exist in different Arab countries and even within the same country, the spoken Tunisian dialect for instance is unlikely to be fully understood in other Arab countries and vice-versa. For example, the classical standard Arabic name for tomorrow is "ghadan", it is pronounced in different dialects throughout the Arab word, such as "bokra" or "bacher". Therefore, often, an effective verbal conversation between people from different Arab countries necessitates the use of the classical Arabic instead of their respective dialect. This is somewhat similar to the American words "faucet, car hood and trunk" compared to the British exact equivalent in "tap, car bonnet and boot". Although the Arabic version of the C-ACT has been validated using the Tunisian Arabic dialect, ${ }^{15}$ the performance of that instrument in other Arabic speaking countries is likely to be very different.

As errors in the management of children with asthma might result from incorrect classification of the level of control derived from the C-ACT questionnaire, it is imperative to analyze the performance of the Arabic version of that instrument in a population that has its own Arabic dialect. We therefore analyzed in Arabic speaking children in the UAE, the correlation between the standard Arabic version of the C-ACT and the GINA assessment of asthma control, as well as with the resulting modifications made in the management of asthma.

\section{RESULTS}

One hundred and five children aged between 4 and 11.8 years (mean age 7 years, 61\% boys) were enrolled in the study. Their anthropometric data, their C-ACT score, their category of GINA

\footnotetext{
${ }^{1}$ Department of Pediatrics, Tawam Hospital in affiliations with Johns Hopkins Medicine, P.O.Box 15258, Abu Dhabi, United Arab Emirates and ${ }^{2}$ Department of Pediatrics, College of Medicine and Health Science, United Arab Emirates University, P.O.Box 17666, Al-Ain, United Arab Emirates

Correspondence: Majid AITeneiji (maltuniji@seha.ae)
}

Received: 23 October 2017 Accepted: 1 October 2018

Published online: 01 November 2018 
Table 1. Sample characteristics of the study population $(n=105)$

Age (y)

Mean \pm SD (median)

$7.9 \pm 2.4(7.8)$

Range

$4.0-11.8$

Anthropometrics

Weight $(\mathrm{kg})$

Height $(\mathrm{cm})$

BMI $\left(\mathrm{kg} / \mathrm{m}^{2}\right)$

$28.5 \pm 11.7$

C-ACT No. (\%)

Controlled

$126.1 \pm 14.5$

$17.2 \pm 4.2$

Uncontrolled

$47(45)$

$58(55)$

Specialist assessment of control using GINA No. (\%)

Well controlled

34 (32)

Partly controlled

Uncontrolled

$48(46)$

Specialist assessed change in therapy No. (\%)

Step down

$2(2)$

No change

$44(42)$

Step up

$B M I$ body mass index, C-ACT Childhood Asthma Control Test, GINA Global Initiative for Asthma

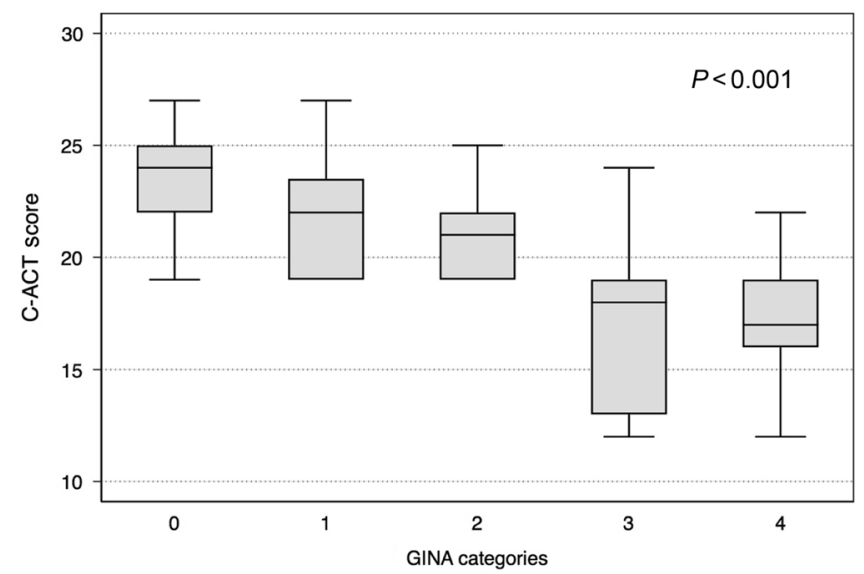

Fig. 1 The C-ACT score correlation with the GINA scoring

assessments of control and the resulting modifications of their asthma management are shown in Table 1.

The Cronbach alpha estimate for the internal consistency or reliability of seven items Arabic C-ACT survey was $0.81 \%$ in the total sample, indicating high consistency and reliability of that tool. The C-ACT score correlated well $(P<0.001)$ with the GINA scoring confirming also its validity (Fig. 1 and Table $2 S$ ). Using a CACT cut-score of 19 to classify the level of control by GINA had a sensitivity of $94.74 \%$, a specificity of $70.83 \%$, a Youden index of 0.65 and correctly classified GINA control in nearly $84 \%$ of the children (Table 2). A C-ACT score of 21 showed the highest Youden index (0.68) with a sensitivity of $80.70 \%$, a specificity of $87.50 \%$ and similarly correctly classified GINA control in nearly $84 \%$ of the children (Table 2 ). The respective receiver operating characteristic (ROC) curve (Fig. 2) yielded an area under curve (AUC) of 0.898 (95\% Cl 0.83-0.96). Using the Youden method the optimal cut-point to classify GINA control categories was a C-ACT score of 20 (standard error $0.8,95 \% \mathrm{Cl} 18.3-21.6, P<0.001$ ).

The C-ACT also correlated well with the resulting change in therapy in 73 participants 70\% (Table 3S). A C-ACT cut-score of 19 had a sensitivity of $78.79 \%$, a specificity of $58.97 \%$, a Youden index

of 0.3776 and correctly classified the treatment change category in $71 \%$ of children, while a score of 21 has a sensitivity of $65.15 \%$, a specificity of $76.92 \%$, a Youden index of 0.42 and correctly classified over $69 \%$ of the children (Table 3). The AUC of the respective ROC curve was 0.749 ( $95 \% \mathrm{Cl} 0.66-0.85$ ) (Fig. 3). The optimal cut-point using the Youden method to classify treatment change categories was a score of 20 (standard error $1.2,95 \% \mathrm{Cl}$ 17.5-22.4, $P<0.001$ ).

The cut-point scores for classifying both the GINA and the treatment control (as well as their respective 95\% confidence intervals) were quasi similar and showed no statistically significant difference $(P=1.0)$, highlighting that a single Arabic C-ACT cutpoint of 20 can be used to adequately classify both levels of control.

\section{DISCUSSION}

Comparing the standard Arabic version of the C-ACT to the GINA assessment of asthma control, we found it to have high reliability as well as validity, as it correlated well with the GINA criteria of asthma control. However, using the recommended cut-point of 19 limit overestimated asthma control in our population. This confirms previous reports where other versions of the C-ACT were used. ${ }^{10,14,16,17}$

We found that a C-ACT score of 20 was optimal to accurately estimate the level of asthma control rather than the recommended traditional cut off limit of 19 in our population. This also validates previous reports which had used the Spanish and French versions $s^{14,16}$ as well as a study from Tunisia which used the Tunisian Arabic dialect version of the C-ACT. ${ }^{15}$ As the majority of previous publications have only reported the Youden index but without its $95 \% \mathrm{Cl}$, it is not possible to statistically compare their optimal score with ours. Other studies, using different languages to English and comparing C-ACT to physician assessment, asthma diaries and/or objectives measures such as spirometry or fractional exhaled nitric oxide, also found that the most appropriate C-ACT cut-offs scores ranged from 20 up to 24 , but unfortunately without providing confidence intervals. ${ }^{10,11,14,16,17,18-22}$ The differences in the cut-scores between GINA and C-ACT are a direct reflection that they are two different tools: while GINA categorizes control based on frequency of symptoms, the C-ACT focuses on their severity. The differences among all the suggested C-ACT cut-off scores by the different studies ${ }^{19-21}$ is probably underpinned by differences in the perception and understanding of asthma control, resulting most likely from differences in cultural and educational backgrounds between the various studied populations. This finding suggests that the proposed cut-off value to define asthma control in the English version of the C-ACT, is different from those where other languages are used.

Although we found a significant correlation between the C-ACT and change in asthma treatment at a cut-point of 19, the Youden index was the highest at a cut-point of 21. However, the computed optimal cut-point was 20, making it therefore more appropriate in our population than the recommended cut-point of 19 , as confirmed in earlier studies. ${ }^{16,18,19,22}$

Interestingly, the cut-point scores for classifying both the GINA and the treatment control were identical, highlighting that a single Arabic C-ACT cut-point of 20 can be used to adequately classify both levels of control, simplifying considerably its use.

One of the limitations in our study was the small sample size. Another was the lack of objective measures to assess asthma control, such as spirometry, although that limitation is probably mitigated by conflicting results of correlation studies between spirometry and asthma control. ${ }^{11,12,23,24}$ Because of the difficultly in using spirometry in young children, there is a need for future studies which will use other objective measures not requiring the child's cooperation such as the forced oscillation technique (FOT). Furthermore, we believe a longitudinal assessment to apply a 
Table 2. Receiver operator characteristic (ROC) curve of C-ACT score performance in classifying GINA categories (well controlled v/s all other categories) in 105 children with asthma

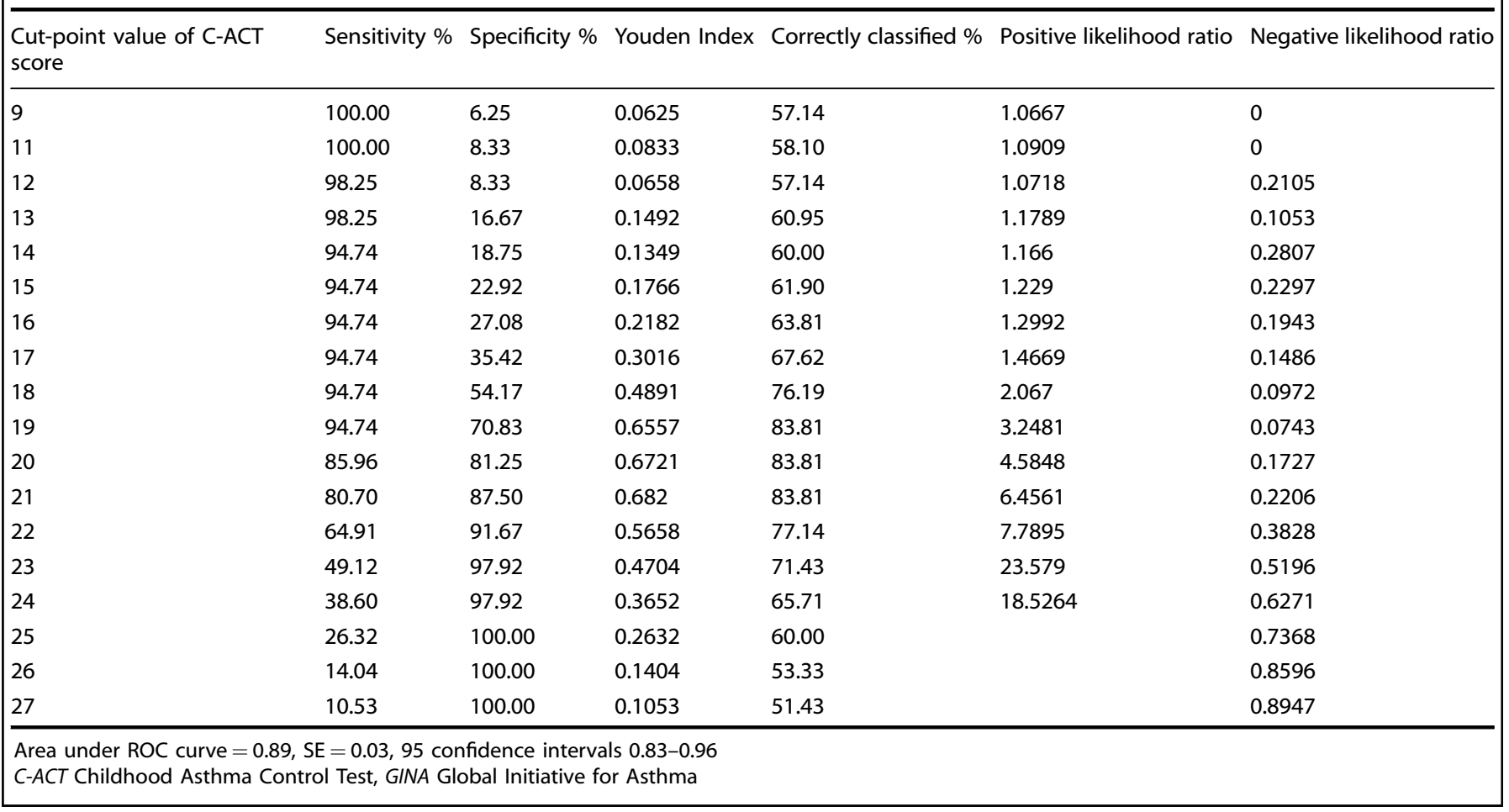

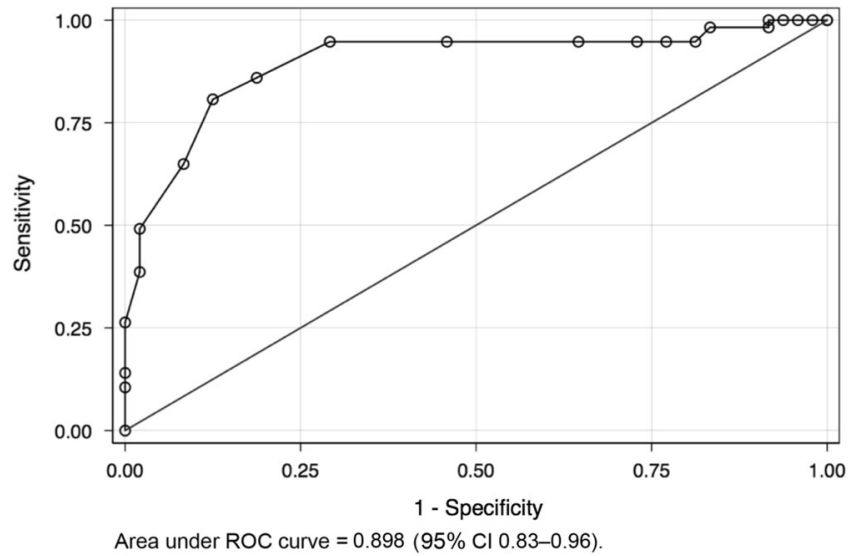

Fig. 2 ROC curve of C-ACT scores by GINA asthma control categories (well controlled versus all other categories)

test-retest analysis will add more strength to future studies. As the majority of previous studies have not calculated or reported their optimal cut-point with $95 \%$ confidence intervals, a formal statistical comparison of the optimal cut-points amongst all the studies was not possible. We encourage future studies to use the methodology which we have used to enable robust comparison of the recommended optimal scores.

The Arabic translated C-ACT had a high reliability and validity. However, the recommended cut-points of the original English version do not accurately define asthma control in native children in the UAE. A single Arabic C-ACT cut-point of 20 correctly classifies the level of control both by GINA or by treatment control, simplifying considerably its use. We believe that, in different populations, especially with different languages, the optimal CACT score cut-point should be specifically calculated to accurately establish asthma control in native children.

\section{METHODS}

\section{Participants}

Emirati national children, aged between 4 and 11 years, having Arabic as their native language, were eligible for enrolment in the study if they had a physician diagnosis of asthma. All study participants were managed by pediatric pulmonology staff in the outpatient pediatric pulmonology clinic at Tawam hospital (Al-Ain city, UAE) between April 2015 until April 2017 and were already on prophylactic therapy for asthma at the discretion of their treating physician.

All participating children had their height and weight measured, and they (or their parents, when appropriate) were requested to complete the Arabic C-ACT questionnaire. The children were then seen by a pediatric pulmonologist who assessed the level of asthma control using the GINA guidelines. ${ }^{5}$ All the described data were obtained in a single visit.

Exclusion criteria includes any systemic illness, seizure disorder, congenital anomaly, cerebral palsy, chest surgery, chronic lung disease of infancy, and upper airway abnormality.

\section{Sample size}

According to our audit results, an estimated $5-10 \%$ of children with asthma (average 7.5\%) attending our clinic are not well controlled. We have therefore calculated that a minimum sample size of 101 participants is required to give the study enough power to detect a $5 \%$ difference in disease control with a precision of 5 and $95 \%$ confidence level. We decided to enroll 105 children to compensate for eventual attrition.

Instrument: Arabic childhood asthma control test

C-ACT is composed of seven questions (four child-reported and three parent-reported) with a score range from zero (poor control) to 27 (complete control). A score $\leq 19$ defines uncontrolled asthma and, when $\leq 12$ the condition is defined as very poorly controlled. ${ }^{8,13}$ The Heath Authority of Abu Dhabi, which governs all government hospitals in the emirate, translated the C-ACT into Arabic, approved the translated tool and recommended its use in all its affiliated health institutions, including ours, in 2014. 
Table 3. Receiver operator characteristic (ROC) curve of C-ACT score performance in classifying treatment change categories (well controlled v/s all other categories) in 105 children with asthma

\begin{tabular}{|c|c|c|c|c|c|c|}
\hline $\begin{array}{l}\text { Cut-point value of C-ACT } \\
\text { score }\end{array}$ & Sensitivity \% & Specificity \% & Youden index & Correctly classified \% & Positive likelihood ratio & Negative likelihood ratio \\
\hline 9 & 98.48 & 5.13 & 0.0361 & 63.81 & 1.0381 & 0.2955 \\
\hline 12 & 96.97 & 7.69 & 0.0466 & 63.81 & 1.0505 & 0.3939 \\
\hline 13 & 93.94 & 12.82 & 0.0676 & 63.81 & 1.0775 & 0.4727 \\
\hline 14 & 92.42 & 17.95 & 0.1037 & 64.76 & 1.1264 & 0.4221 \\
\hline 16 & 90.91 & 25.64 & 0.1655 & 66.67 & 1.2226 & 0.3545 \\
\hline 17 & 86.36 & 28.21 & 0.1457 & 64.76 & 1.2029 & 0.4835 \\
\hline 18 & 84.85 & 48.72 & 0.3357 & 71.43 & 1.6545 & 0.311 \\
\hline 19 & 78.79 & 58.97 & 0.3776 & 71.43 & 1.9205 & 0.3597 \\
\hline 20 & 69.70 & 69.23 & 0.3893 & 69.52 & 2.2652 & 0.4377 \\
\hline 25 & 21.21 & 97.44 & 0.1865 & 49.52 & 8.2727 & 0.8086 \\
\hline 26 & 10.61 & 97.44 & 0.0805 & 42.86 & 4.1364 & 0.9175 \\
\hline 27 & 7.58 & 97.44 & 0.0502 & 40.95 & 2.9545 & 0.9486 \\
\hline
\end{tabular}

Area under ROC curve $=0.75, \mathrm{SE}=0.05,95$ confidence intervals $0.65-0.84$

C-ACT Childhood Asthma Control Test

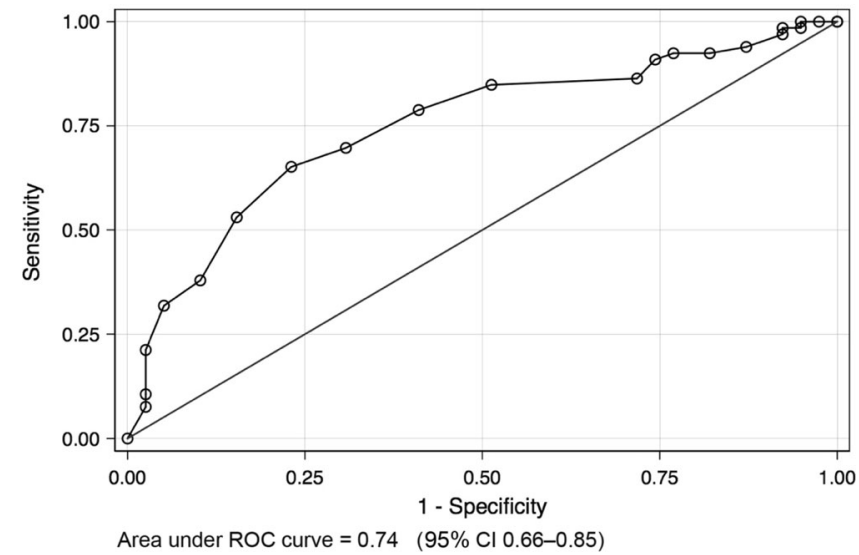

Fig. 3 ROC curve of C-ACT scores by treatment change categories (well controlled versus all other categories)

GINA assessment of asthma control

In GINA assessment of asthma control the physician evaluates asthma control by assessing the presence of the following symptoms in the 4 preceding weeks: (1) presence of daytime asthma symptoms, (2) nighttime asthma symptoms, (3) activity limitation and (4) use of short acting $\beta 2$ agonist. The resulting level of asthma control is classified as (1) well controlled if the child does not have any of the above symptoms, (2) partially controlled in the presence of one or two of the above symptoms and (3) uncontrolled in the presence of at least three of the above symptoms. ${ }^{5}$

\section{Change in therapy based on physician assessment of asthma control}

Using their respective physician's discretion to modify the therapeutic plan, according to the physician assessment, the participants were categorized into three groups: (1) step down (reducing inhaled steroid dose/long acting beta agonists (LABA) or Montelukast), (2) no modification to the management, and (3) step up in therapy, consisting of either (a) increasing the dose of inhaled steroid dose or the addition of LABA or Montelukast if the children were deemed to be uncontrolled, or (b) maintaining the same medication if the participants were deemed to be non-compliant or if their medication administration technique was suboptimal. For the analysis, the number of categories was reduced to only two: (1) controlled group (if medications were stepped down or not changed) and (2) uncontrolled group (if medications were stepped up).

Ethics approval

The study was approved by the institutional research review board (ref: CRD 531/17 -AAMDHREC Protocol No. 531-17). All research was conducted in accordance with all relevant guidelines and procedures.

Statistical analysis

The reliability, or internal consistency of the seven items on C-ACT was assessed by the Cronbach's alpha test. Its validity was evaluated by the degree of correlation it had with the score of the GINA assessment of asthma control.

Normally distributed continuous variables were reported as mean \pm standard deviation (SD) and categories distribution as number of participants (and percentage).

The univariate analysis of continuous variables (C-ACT and GINA) between the categories was conducted with the Analysis of Variance test (ANOVA). Proportions were compared with the $x^{2}$ test and the Fisher exact test was used for small proportions.

The sensitivity, specificity, Youden index, percentage of correct classification, positive and negative likelihood ratios were calculated for the different cut-scores of the C-ACT to categorize the GINA assessment as well as the categorization of the change in asthma management. The resulting receiver operator characteristic (ROC) curves were constructed and their respective area under the ROC curve (AUC), with 95\% confidence intervals $(\mathrm{Cl})$, were reported. In addition, using the Youden method, the optimal cut-score (with $95 \% \mathrm{Cl}$ ) was computed using bootstrapping with 100 replications.

All the analyses were performed with the STATA statistical package version 14 (StataCorp, College Station, TX, USA) and statistical significance was defined by a two-sided $p$-value $<0.05$. 


\section{DATA AVAILABILITY}

All data generated or analyzed during this study are included in this published article (and its supplementary information files).

\section{ACKNOWLEDGEMENTS}

We are grateful to all participating children, their families and the physicians who have facilitated their enrolment.

\section{AUTHOR CONTRIBUTIONS}

M.A. conceived the study and was the primary investigator. All authors researched, collated, and wrote this paper.

\section{ADDITIONAL INFORMATION}

Supplementary information accompanies the paper on the npj Primary Care Respiratory Medicine website (https://doi.org/10.1038/s41533-018-0109-3).

Competing interests: The authors declare no competing interests.

Publisher's note: Springer Nature remains neutral with regard to jurisdictional claims in published maps and institutional affiliations.

\section{REFERENCES}

1. World Health Organization. Asthma Fact Sheet, <http://www.who.int/ mediacentre/factsheets/fs307/en/>(2017).

2. Al-Maskari, F. et al. Asthma and respiratory symptoms among school children in United Arab Emirates. Allerg. Immunol. 32, 159-163 (2000).

3. Masoli, M., Fabian, D., Holt, S. \& Beasley, R. The global burden of asthma: executive summary of the GINA Dissemination Committee report. Allergy 59, 469-478 (2004)

4. Bener, A., Abdulrazzaq, Y. M., Debuse, P. \& Abdin, A. Asthma and wheezing as the cause of school absence. J. Asthma 31, 93-98 (1994).

5. Global Initiative for Asthma - GINA. (2017). Global Initiative for Asthma - Global Initiative for Asthma -GINA. [online] Available at: https://ginasthma.org/ [Accessed 5 May 2017].

6. Carroll, W., Wildhaber, J. \& Brand, P. Parent misperception of control in childhood/ adolescent asthma: the Room to Breathe survey. Eur. Respir. J. 39, 90-96 (2012)

7. Taylor, D. et al. A new perspective on concepts of asthma severity and control. Eur. Respir. J. 32, 545-554 (2008).

8. Liu, A. H. et al. Development and cross-sectional validation of the Childhood Asthma Control Test. J. Allergy Clin. Immunol. 119, 817-825 (2007).

9. Waibel, V., Ulmer, H. \& Horak, E. Assessing asthma control: symptom scores, GINA levels of asthma control, lung function, and exhaled nitric oxide. Pediatr. Pulmonol. 47, 113-118 (2012).

10. Erkocoglu, M. et al. Consistency of GINA criteria and childhood asthma control test on the determination of asthma control. Pediatr. Allergy Immunol. 23, 34-39 (2012).
11. Chen, H.-H., Wang, J.-Y., Jan, R.-L., Liu, Y.-H. \& Liu, L.-F. Reliability and validity of childhood asthma control test in a population of Chinese asthmatic children Qual. Life Res. 17, 585-593 (2008).

12. Sekerel, B. et al. The reliability and validity of Turkish version of Childhood Asthma Control Test. Qual. Life Res. 21, 685-690 (2012).

13. Liu, A. H. et al. The Childhood Asthma Control Test*: retrospective determination and clinical validation of a cut point to identify children with very poorly controlled asthma. J. Allergy Clin. Immunol. 126, 267-273. e261 (2010).

14. Pérez-Yarza, E. et al. Validation of a Spanish version of the childhood asthma control test (SC-ACT) for use in Spain. An. De. Pedia. 83, 94-103 (2015).

15. Ouali, $\mathrm{H}$. et al. Childhood asthma control test: validation of the Arabic Tunisian dialect version in 51 patients. Eur. Respir. J. 40, P3970 (2012).

16. Deschildre, A. et al. Asthma control assessment in a pediatric population: comparison between GINA/NAEPP guidelines, Childhood Asthma Control Test (CACT), and physician's rating. Allergy 69, 784-790 (2014).

17. Koolen, B. B. et al. Comparing Global Initiative for Asthma (GINA) criteria with the Childhood Asthma Control Test (C-ACT) and Asthma Control Test (ACT). Eur. Respir. J. 38, 561-566 (2011).

18. Ito, Y. et al. Association between the results of the childhood asthma control test and objective parameters in asthmatic children. J. Asthma 48, 1076-1080 (2011).

19. Voorend-van Bergen, S. et al. Monitoring childhood asthma: web-based diaries and the asthma control test. J. Allergy Clin. Immunol. 133, 1599-1605.e1592 (2014).

20. Yavuz, S. T. et al. Identifying uncontrolled asthma in children with the childhood asthma control test or exhaled nitric oxide measurement. Ann. Allergy, Asthma Immunol. 109, 36-40 (2012).

21. Leung, T. et al. Identifying uncontrolled asthma in young children: clinical scores or objective variables? J. Asthma 46, 130-135 (2009).

22. Shi, Y., Tatavoosian, A. V., Aledia, A. S., George, S. C. \& Galant, S. P. Cut points for Asthma Control Tests in Mexican children in Orange County, California. Ann Allergy, Asthma Immunol. 109, 108-113 (2012).

23. Bacharier, L. B. et al. Classifying asthma severity in children: mismatch between symptoms, medication use, and lung function. Am. J. Respir. Crit. Care Med. 170 426-432 (2004)

24. Muiño, A., Torello, P. \& Brea, S. Test de control de asma en pediatría: ACT infantil Utilidad clínica en la práctica diaria. Arch. De. Pedia. Del. Urug. 81, 78-86 (2010).

Open Access This article is licensed under a Creative Commons Attribution 4.0 International License, which permits use, sharing, adaptation, distribution and reproduction in any medium or format, as long as you give appropriate credit to the original author(s) and the source, provide a link to the Creative Commons license, and indicate if changes were made. The images or other third party material in this article are included in the article's Creative Commons license, unless indicated otherwise in a credit line to the material. If material is not included in the article's Creative Commons license and your intended use is not permitted by statutory regulation or exceeds the permitted use, you will need to obtain permission directly from the copyright holder. To view a copy of this license, visit http://creativecommons. org/licenses/by/4.0/.

(c) The Author(s) 2018 